\title{
Cutaneous and renal glomerular vasculopathy
}
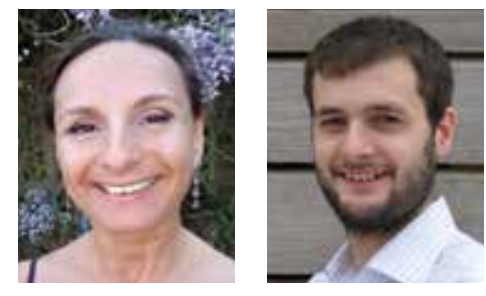

Laura Holm and David Walker, of Anderson Moores

\section{Veterinary}

Specialists, update

Companion

readers as to our

current knowledge

of CRGV and

introduce their new

research project

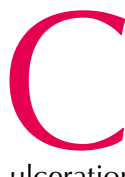

utaneous and renal glomerular vasculopathy (CRGV or Alabama rot) is a disease of currently unknown cause characterized by ulceration of the distal extremities in dogs and variably associated with acute kidney injury (AKI). CRGV is causing increasing concern to dog owners and veterinary professionals across the UK.

Since November 2012, more than sixty dogs across the UK have been identified with clinicopathological findings similar to those reported in a 1980's case series which first described CRGV in US Greyhounds. Skin lesions, typically appearing as ulcers or erosions on the distal limbs, ventrum or oral cavity/muzzle, commonly appear less than a week before clinical signs attributable to AKI.

\section{Decision making}

Data from previous studies in greyhounds with presumed CRGV suggests that up to $75 \%$ of cases develop skin lesions without clinically apparent AKI, and that the prognosis for these dogs appears favourable. Even in the absence of azotaemia, renal function may not be normal in these cases and there is some evidence to suggest a proportion of these dogs have pre-azotaemic renal injury. Glomerular filtration rate (GFR) measurement can identify reduced renal function prior to the development of azotaemia and early measurement of GFR in dogs presenting with skin lesions suggestive for CRGV may help with clinical decision making.

A range of breeds have been identified with CRGV in the UK, suggesting that the disease does not solely affect greyhounds. There does not appear to be a breed, body weight, sex or age predilection. Cases have been identified across the whole of the UK and there may be a seasonal distribution with cases being identified between November and June.

\section{Understanding the disease}

The major renal histopathological lesion reported in CRGV is thrombotic microangiopathy (TMA). Thrombotic microangiopathies are characterized by inflammation and damage to vascular endothelium. This is thought to lead to platelet activation and widespread formation of microthrombi, with resultant consumptive thrombocytopenia and microangiopathic haemolytic anaemia. Thrombotic microangiopathies also occur in people and often result in acute kidney injury, thrombocytopaenia and anaemia. Skin lesions have occasionally been reported with some of the diseases that result in TMAs in humans.

Thrombocytopenia has been identified in approximately half of the dogs affected by CRGV and is thought to be the result of platelet consumption. Anaemia, neutrophilia and hyperbilirubinaemia have also been seen in some affected dogs. Approximately 25\% of dogs with CRGV are azotaemic at the time of first presentation; however, most appear to develop azotaemia a few days after initial presentation with a skin lesion(s) (median 3 days, range $1-10$ days).

Aside from the renal cortices appearing hyperechoic, abdominal ultrasonography has been largely unremarkable in dogs with CRGV. Hypertension is possible, as it is in any patient with AKI.
A definitive diagnosis of CRGV is made by histopathological assessment of the kidneys and, where possible, skin. The aetiology of CRGV remains unknown at this time and common causes of AKI should be considered and excluded when presented with a dog that may be suffering from CRGV.

Current knowledge suggests that management should be as for any other cause of AKI; however, specialist advice should be sought with a patient with suspected CRGV. Even in people, diseases resulting in TMA are still relatively poorly understood. Many treatment options exist; however, the optimum treatment often remains unknown and treatment is not always successful.

The prognosis for dogs with CRGV and clinically significant AKI appears to be poor, although with intensive management some dogs have survived and regained normal renal function. The question remains as to whether CRGV is an emerging disease or one that was previously unrecognized. C

\section{PARTICIPATE IN THE RESEARCH}

It can be very challenging to know if an erosive or ulcerative skin lesion of unknown cause is the result of CRGV or something else, as azotaemia is typically absent at initial presentation and urine concentrating ability may be retained. Glomerular filtration rate (GFR) measurement may allow early detection of pre-azotaemic AKI and thus direct appropriate management.

To investigate further, David and Laura alongside their colleagues Rosanne Jepson and Ludovic Pelligand at the Royal Veterinary College (RVC) - are inviting veterinary surgeons presented with a dog with a skin lesion(s) of unknown cause to contact Anderson Moores Veterinary Specialists (medicine@andersonmoores.com; 01962 767920) to discuss GFR measurement. Samples can be collected in your practice and laboratory testing performed at the RVC; results will be available within 24 -hours, thus aiding clinical decision making. Aside from blood sampling, all costs will be covered. 